\title{
An analysis of auditory alphabet confusions
}

\author{
MICHAEL E. WALKER \\ University of Illinois, Urbana-Champaign, Champaign, Illinois
}

\begin{abstract}
The present study, using the nonhierarchical overlapping clustering algorithm MAPCLUS to fit the Shepard-Arabie (1979) ADCLUS model, attempted to derive a set of features that would accurately describe the auditory alphabet confusions present in the data matrices of Conrad (1964) and Hull (1973). Separate nine-cluster solutions accounted for $80 \%$ and $89 \%$ of the variance in the matrices, respectively. The clusters revealed that the most frequently confused letter names contained common vowels and phonetically similar consonants. Further analyses using INDCLUS, an individual differences extension of the MAPCLUS algorithm and ADCLUS model, indicated that while the patterns of errors in the two matrices were remarkably similar, some differences were also apparent. These differences reflected the differing amounts of background noise present in the two studies.
\end{abstract}

A widely used methodology in the study of speech perception involves presenting subjects with a random ordering of phonemes. The subjects are then asked, for each stimulus, to report the phoneme they think was presented. The data from such a study are conveniently arranged in an $N \times N$ array, with the entries consisting of the number of times the row stimulus was identified as the column stimulus. The resulting confusion matrix may be constructed without any knowledge of the physical attributes of the stimulus; instead, its structure reflects the patterns of similarities of the psychological attributes of the stimuli as perceived by the listener (Shepard, 1972). Once these psychological characteristics are identified, one can attempt to discover their relationship to the purely physical structure of the phonemes (Kewley-Port, 1983).

The analysis of confusion matrices typically involves the specification of a set of distinctive features, each containing mutually exclusive categories, by which the different stimuli may be classified. The relative importance of these features as reflected in some performance measure (e.g., percent of information transmitted or error rate) is then determined (Wang \& Bilger, 1973). Most often, in the case of auditory stimuli, articulatory features such as place or manner make up these systems (e.g., Miller \& Nicely, 1955; Wickelgren, 1966).

Researchers have taken diverse approaches to the evaluation of features as accurate descriptors of the inherent structure in the data. Wickelgren (1966), in his comparison of three feature systems, rank-ordered the response

The research reported here was conducted while the author held a CIC Minorities Fellowship. This research was supported in part by both NSF Grant SES 8310866 and the AT\&T Industrial Affiliates Program at the University of Illinois (Phipps Arabie, Principal Investigator). The author wishes to acknowledge Phipps Arabie, whose comments and technical assistance proved invaluable in the preparation of this manuscript. The author also thanks the anonymous reviewers for their comments. Requests for reprints should be addressed to Michael E. Walker, Department of Psychology, University of Illinois, 603 East Daniel St., Champaign, IL 61820 . phonemes for each stimulus by using the frequency of their occurrence. Then, for pairs of responses, he calculated the number of directional predictions made by each feature, as well as the percentage of these predictions found in the data. Finally, he compared the overall relative performance of the three feature systems by means of a chisquare test statistic.

Miller and Nicely's (1955) work focused more upon the performance of the individual features. For each feature, the researchers computed articulation scores by dividing the consonant phonemes into categories and computing the ratio of the number of intracategory correct responses to the total number of responses. Using this method, Miller and Nicely were able to ascertain the relative importance of each feature in describing the data. Hubert and Baker (1977) devised an index of goodnessof-fit of individual features on the basis of the averaged within-group errors; they estimated the significance level of the index for each feature by using a permutation test based upon 100 random partitions of the same form (i.e., with subsets of the same size) as the partition for the feature. The use of this procedure to describe the data yielded an objective index of the absolute importance of each feature, considered individually.

A shortcoming of the procedures used by Miller and Nicely (1955) and by Hubert and Baker (1977) is a failure to correct for the lack of independence among the features used. That is, some overlap may exist in the actual information that two features explain. Klatt (1968) used Miller and Nicely's approach to divide the phonemes from Wickelgren's (1966) study. For each feature, he computed the number of cross-set confusions and subtracted it from its expected value (assuming independence) based on row and column sums. To derive an index summarizing the evaluation of the feature, this quantity was divided by a standard deviation estimated from the Bernoulli nature of the experiment. By using a sequential process in which the feature with the highest index at each stage was partialed out of each of the remaining features, Klatt de- 
rived the relative performance of the features while taking into account their interdependence. Wang and Bilger's (1973) sequential information analysis (SINFA) was similar to Klatt's stepwise procedure in that it partialed out the effects of previously analyzed features. Instead of the index used by Klatt, however, Wang and Bilger used the amount of information transmitted as their measure of the importance of a feature in the set.

All of the above methods require that the researcher specify the categories into which the stimuli will be placed (Soli, Arabie, \& Carroll, 1986). Another approach would be an exploratory one in which a set of features is derived from the structure of the data. One widely used exploratory technique has been multidimensional scaling (MDS), by which an attempt is made to position the stimuli in an $n$-dimensional psychological space. Shepard (1972), using his specially developed MDS program, which fitted an exponential decay function (Chang \& Shepard, 1966), reanalyzed the Miller-Nicely (1955) data and found that the procedure separated the unvoiced stops and fricatives, the voiced stops and fricatives, and the nasals along dimensions which he labeled voicing and nasality.

There is some question as to whether, in general, spatial interpretations are valid for phoneme confusions. The assumptions of continuity inherent in MDS methods with respect to the stimulus attributes of confusions and their influence on the perceptual similarity of the stimuli have been questioned (Soli et al., 1986). Many believe that the underlying psychological characterization of phonemes is not continuous. Rather, it is viewed as a discrete one (Shepard, 1972, 1974; Shepard \& Arabie, 1979; Stevens, 1972). In this case, continuous scaling methods may be deemed inappropriate.

An alternative to continuous methods of data analysis is found in the various hierarchical clustering procedures, which offer a discrete solution without the constraint of the above feature-analytic methods of a priori specification of the stimulus categories. In general, these methods attempt to create subsets or clusters of the original stimuli in such a way that the intracluster similarities exceed some minimum criterion. In particular, the derived similarities among the stimuli are transformed to compatibility with the ultrametric inequality (Johnson, 1967). Johnson and, later, Shepard (1972) applied hierarchical clustering techniques to the Miller-Nicely (1955) data. At the level of five clusters, Johnson was able to identify the clusters using the descriptive feature system of Miller and Nicely. Shepard reported similarly interpretable clusters in his reanalysis of the data.

A strong limitation of hierarchical clustering is that for any given distinct pair of clusters, either one is a proper subset of the other, or they are mutually exclusive. The algorithms for such clustering do not allow for the possibility of overlapping (but nonhierarchical) clusters, and thus greatly limit the admissible structure (cf. Soli et al., 1986). Shepard and Arabie (1979) developed a nonhierarchical additive clustering (ADCLUS) model which allowed clusters to overlap. In the model, similarity between any two objects is seen as an additive function of the discrete, weighted attributes common to both objects. Using the MAPCLUS algorithm for fitting the ADCLUS model, Arabie and Carroll (1980b) obtained eight fairly interpretable clusters that accounted for almost $90 \%$ of the variance in the Miller-Nicely data.

The present study is concerned with the alphabetic confusion matrices of Conrad (1964) and Hull (1973). Unlike the matrices in the above studies, which involve confusions among phonemes, the matrices of Hull and of Conrad represent confusions among letter names (e.g., "ay," "bee," "see”). As letter names usually consist of more than one phoneme, one would expect that the determinants of confusions among them would be more complex than in the single-phoneme case. A plausible hypothesis is that the confusion between any two letters would be mediated by the features of the individual phonemes of which they are composed, with an increase in the features shared by the two letters leading to a corresponding increase in the confusability between them.

Wickelgren (1965), in a study of short-term memory for letters and digits, noted that the letters that are confused most often have at least one phoneme in common. Morgan (1973) reported that the second phoneme seems to be more important in determining the pattern of confusions than is the first. His single-link cluster analysis of Conrad's (1964) data, however, resulted in clusters not readily interpretable when using phonetic features. One possible reason could be the use of single-link clustering, which exhibits some undesirable properties (Lance \& Williams, 1967). Morgan tested for this possibility by applying Jardine and Sibson's (1968) $\mathrm{B}(k)$, an extension of single-link clustering, to Conrad's data. When $k$ is set equal to 1 , the method is identical to single-link clustering. When $k$ is greater than 1 , the method is a nonhierarchical overlapping approach that allows for $k-1$ elements in the intersection of any two clusters. Morgan used $B(2)$ which, while disrupting the chaining effects of singlelink clustering, still did not lead to easily interpretable clusters. This finding could have indicated that the value of $k$ was not sufficiently great to counteract the distorting effects of the single-link algorithm.

The purpose of the present study was to determine if a set of acoustic features could be found which would reflect the patterns of confusions in the Hull (1973) and Conrad (1964) data sets. The study also tested the generalizability of the derived feature sets from one data matrix to the other. The MAPCLUS algorithm is ideal for this purpose, because it measures the extent to which an a priori set of features explains a data set in terms of variance accounted for. It also derives a set of clusters that are not limited by the constraints of strict hierarchy and all-ornone overlap.

\section{METHOD}

\section{Experimental Data}

The data from Hull's (1973) experiment consist of identification confusions among 26 letters (with $O$ being simultaneously a letter 
and a digit) and the nine digits 1 to 9 . Six trained speakers, 3 male and 3 female, pronounced randomized orders of the 35 stimuli at the rate of one every $5 \mathrm{sec}$, speaking with standard British pronunciation ( $Z$ is pronounced as "zed," not "zee"). The sound levels of the voices were adjusted to the same mean value, and sufficient white noise was added to bring the signal-to-noise ratio to $0 \mathrm{~dB}$. All six lists were administered to each of 135 subjects. After hearing each stimulus, the subjects recorded the letter that they believed had been presented. Results for all six lists were pooled. Confusions among only the 26 letters of the alphabet were used in the present study. The error rate for the reduced matrix was $40 \%$.

In Conrad's (1964) study, the stimuli consisted of the 26 letters of the alphabet, read in random order by 10 untrained British speakers, 6 male and 4 female, at the rate of one letter every $5 \mathrm{sec}$. An (on average) equal amount of white noise was added to the stimuli. Each of the 300 subjects listened to five repetitions of the alphabet spoken by 5 male and 2 female voices. The subjects recorded their responses in the manner described above. Responses of all subjects were pooled, and the overall error rate was $61 \%$.

\section{Symmetrization of Confusion Data}

The ADCLUS model (described below in detail) assumes that the input matrix of similarities is symmetric. The two confusion matrices used here are not strictly symmetric. Hubert and Baker (1979) have devised a method for assessing the extent to which a matrix departs from symmetry. They compute an R-index, which is in fact equivalent to the product-moment correlation between the corresponding off-diagonal entries in the matrix and its transpose. A positive value of $R$ indicates a symmetric matrix, and a negative value of $R$ indicates a skew-symmetric matrix. This value of $R$ is compared with the distribution of $R$-indices obtained by randomly permuting the columns and rows of the matrix. The $R$-indices for the two data matrices in the present study are .413 for the Hull matrix and .615 for Conrad's. Based upon the estimated reference distributions obtained as described above, the null hypothesis of a random matching may be rejected in each case, with an estimated significance level of .001 for both matrices. There does not appear to be any systematic nonsymmetry in the data. While symmetrization of the data does lose some information, it does not appear, in this case, to violate their underlying structure.

Many methods for symmetrizing data have been proposed (Arabie \& Soli, 1982, list four commonly used formulas). The symmetrization method used here was an arithmetic mean of the corresponding off-diagonal elements, where the $i j$ th entry in the symmetrized matrix is equal to half the sum of the $i j$ th and $j i$ th entries of the original matrix. This value is perhaps the simplest measure to compute, and, although it has the disadvantage of not taking into account the main diagonal elements, research has shown that solutions obtained using various symmetrization methods are empirically often very similar (Shepard, 1972; Van Der Kamp \& Pols, 1971).

\section{The MAPCLUS Algorithm}

The MAPCLUS program fits the ADCLUS model described by Shepard and Arabie (1979). Formally, the model reconstructs the matrix of interstimulus similarity by the formula

$$
\mathbf{S}=\mathbf{P} \mathbf{W} \mathbf{P}^{\prime},
$$

where $\mathrm{S}$ is the $N \times N$ symmetric matrix of predicted similarities among $N$ objects, $\mathbf{W}$ is a $K \times K$ diagonal matrix of weights associated with $K$ clusters, and $\mathrm{P}$ is an $N \times K$ rectangular matrix of binary values. The weight associated with a given cluster indicates its relative contribution toward explaining the pattern of similarities in the data. Each $i k$ th entry in $P$ assumes a value of 1 if stimulus $i$ belongs to subset $k$, and 0 otherwise. The model adds a matrix $\mathbf{C}$ with zeroes on the main diagonal and the additive constant $c$ in the off-diagonals. This constant may be interpreted as the weight fitted for the complete set of stimuli, and is necessary for the computation of variance accounted for in assessing goodness-of-fit.
The MAPCLUS algorithm and its implementation are described in detail by Arabie and Carroll (1980a, 1980b). In the initial stages of computation, the entries in the matrix $\mathrm{P}$ are not restricted to binary values. Briefly, MAPCLUS fits Equation 1 by simultaneously maximizing the variance accounted for (VAF) in the original data, and moving the entries in $\mathbf{P}$ toward 0,1 . The relative importance of these two objectives is systematically varied throughout the course of the computations. The algorithm proceeds in four distinct stages: The first is an iterative approach which maximizes the variance accounted for by the subsets in P; the second and third stages act to refine the fit, using an alternating least squares approach (Carroll \& Chang, 1970; Wold, 1966); and the last stage is a combinatorial optimization procedure that attempts to increase the VAF further.

\section{The INDCLUS Algorithm}

The INDCLUS program (Arabie, Carroll, \& DeSarbo, 1987; Carroll \& Arabie, 1982, 1983) fits an individual differences generalization of the ADCLUS model. The model may be written

$$
\mathbf{S}^{h}=\mathbf{P} \mathbf{W}^{\boldsymbol{h}} \mathbf{P}^{\prime}
$$

where $\mathbf{S}^{n}$ is an $N \times N$ matrix of predicted similarities for subject (or other data source) $h, \mathbf{W}^{h}$ is a $K \times K$ diagonal matrix of weights associated with $K$ clusters for subject $h$, and $P$ is an $N \times K$ matrix of binary values. In the INDCLUS model, subjects are assumed to weight differentially a set of common clusters. The program allows the user to specify that the set of $h$ input matrices are either unconditional (i.e., the matrices are considered to be "all on the same scale") or conditional (i.e., the program separately normalizes the matrices to have unit variance, as in the INDSCAL program of Carroll \& Chang, 1970). The implementation of the INDCLUS program constitutes a generalization of MAPCLUS.

\section{Initial Configuration}

MAPCLUS includes an option to allow the user to specify the initial subset matrix $\mathbf{P}$; he or she may then request the full iterative procedure or, alternatively, that only the weights $(W)$ and VAF be computed by the program. This form of constrained analysis is sometimes referred to as "stand-alone regression" in the documentation (Arabie \& Carroll, 1980a). To test how well acoustical features of individual phonemes explain the data in the present study, a feature list based upon that of Wickelgren (1965) was input into the program, thus defining the "clusters" embodied in the matrix P. Because the algorithm accepts only binary values, Wickelgren's six-category feature of openness was dummy-coded to yield 5 binary features: stops and affricates, fricatives, semivowels and liquids, high vowels, and low vowels. Similarly, the 3 features labial, alveolar, and palatal consonants replaced Wickelgren's fourcategory feature of place. Voiced and nasal were retained from the feature system. The category of front vowels is the only addition to Wickelgren's feature system. Thus, an 11-feature set was defined. For the initial analyses, the alphabet letters were categorized according to their component phonemes, so that the letter $K$ (phonetically, $/ \mathrm{ke} /$ ), for example, would be classified as both a stop (for the initial $/ \mathrm{k} /$ ) and as a front vowel (for the vowel /e/).

\section{Procedure}

The data were analyzed in three separate stages. In the first stage a "stand-alone regression" was implemented for Hull's data using Wickelgren's feature system as the initial configuration (i.e., letting that system define $\mathbf{P}$ in Equation 1). This mode of the MAPCLUS program yielded the variance accounted for by the feature set, as well as the relative importance, or salience, of each feature. In addition, to uncover any other viable features that would explain the data set, MAPCLUS was allowed to search for an optimum 11-cluster solution using the (iterative) alternating least squares procedure. To determine whether solutions containing more or fewer than 11 clusters would prove more interpretable or give improved fit, or both, 8- and 12-cluster solutions were generated. 
In the second stage of the analysis, the features derived in Stage 1 were used as the initial configuration for an analysis of Conrad's data. Again, stand-alone regression was performed to assess the adequacy of the new features (according to the ADCLUS model) in describing the patterns inherent in the data.

The third stage simultaneously analyzed the two matrices using INDCLUS. We sought a solution that would point out the similarities and the differences between the types of confusions in the two matrices. The features derived in the first stage (for the Hull data) were used as the initial configuration for this iterative analysis.

\section{RESULTS AND DISCUSSION}

The stand-alone regression on the Hull data, using Wickelgren's feature list, showed that the total variance explained by the features was only $32.9 \%$, much less than what would intuitively be hoped. The weights indicated that the only cluster to make a large contribution to the overall VAF was that consisting of the nasals $\mathrm{M}$ and $\mathrm{N}$. The second most heavily weighted cluster, corresponding to letters containing the high vowels, was of some importance. The rest of the clusters received near-zero weighting, indicating little or no explanatory power.

Because Wickelgren's feature list did not explain much of the variance in Hull's data, the MAPCLUS program was allowed to generate optimal 8-, 11-, and 12-cluster solutions, beginning with random initial configurations. The variance accounted for by each of the three solutions was $86 \%, 93 \%$, and $96 \%$, respectively. All three solutions revealed several common clusters. The 11- and 12cluster solutions included only one interpretable cluster that was not found in the 8-cluster solution. Augmenting the 8-cluster solution with this additional subset produced a 9-cluster solution that had VAF nearly identical to the best such solution resulting from straightforward use of MAPCLUS, but with superior interpretability. This procedure has been justified by Shepard and Arabie (1979, p. 110) and Arabie and Carroll (1980b, pp. 223, 227), with evaluation of the (statistical) goodness-of-fit showing negligible decrease while substantive utility is markedly increased. The resulting feature list is shown in Table 1. It is identical, except for Cluster 5, to the most interpretable 8-cluster solution. The table also lists the results of a stand-alone regression using the Hull data. As can be seen, the total VAF remained high for the solution, at just over $89 \%$.

The first five clusters were letter dyads. The most heavily weighted of these $(A, K)$ contained letters ending in /e/ and (in the case of $K$ ) containing unvoiced stop consonants. The second cluster was the nasals $(M, N)$. These also shared a common vowel sound. The next two clusters both contained fricatives, and each contained the letter $S$. It is interesting that they formed two separate clusters: The letters $F$ and $S$ are both continuants, while $S$ and $X$ differ only in the inclusion in the latter of the stop $/ \mathrm{k} /$. Similarly, Cluster 5 elements $(\mathrm{Q}, \mathrm{U})$ differed only in the stop $/ \mathrm{k} /$. Clusters 6 and 7 are interesting in that the letter $\mathrm{D}$ appeared in the cluster consisting of the labials and not in the cluster consisting of the alveolars.

A major reason for the inclusion of D in Cluster 6 was its large confusability with $E$ and $B$. Confusion of $D$ with $B$ is understandable in light of the fact that both letters contain voiced stops. The letters $C$ and $T$, on the other hand, both contain unvoiced consonants. Voicing in this instance played a more important role than did place of articulation. Nevertheless, the overall composition of the two clusters fostered labeling them using place of articulation. Cluster 8 contained all of the letters with stops followed by /i/, as well as the letter C. Cluster 9 contained the sonorant consonants with high vowels, except for $\mathrm{W}$.

To test the generalizability of the new feature list, a stand-alone regression was performed on the Conrad (1964) data, using the new feature list as the initial configuration. The results of this analysis are shown in Table 1 . The total VAF of $60 \%$ was much lower than had been expected. As can be seen, the first three clusters still had fairly large weights for the Conrad data; however, the sixth through the ninth clusters had low weights. The poorer performance of Clusters 8 and 9 was to be expected given their low weightings when applied to the Hull data. However, the near-zero weight of Cluster $5(Q, U)$, in-

Table 1

Derived 9-Cluster Solution for the Hull (1973) Data,

With Clusters Ranked According to Numerical Weight

\begin{tabular}{|c|c|c|c|c|}
\hline \multirow[b]{2}{*}{ Cluster } & \multicolumn{2}{|c|}{ Weight } & \multirow[b]{2}{*}{ Constituents } & \multirow[b]{2}{*}{ Interpretation } \\
\hline & $\begin{array}{l}\text { Hull } \\
\text { Data }\end{array}$ & $\begin{array}{c}\text { Conrad } \\
\text { Data }\end{array}$ & & \\
\hline 1 & .990 & .239 & A $\mathrm{K}$ & Unvoiced continuants ending in /e/ \\
\hline 2 & .768 & .948 & M N & Nasals \\
\hline 3 & .754 & .922 & F S & Unvoiced continuants following $/ \varepsilon /$ \\
\hline 4 & .590 & .628 & S X & Sibilants following $/ \varepsilon /$ \\
\hline 5 & .475 & .002 & $\mathbf{Q} \mathbf{U}$ & Containing / $\mathrm{u} /$ (except $\mathrm{W})$ \\
\hline 6 & .359 & .147 & $\mathrm{CT}$ & Alveolars ending in /i/ (except D) \\
\hline 7 & .304 & .118 & B D E P V & Labials ending in /i/ and D \\
\hline 8 & .115 & .125 & B C D E G P T & Stops ending in $/ \mathrm{i} /$ and $\mathrm{C}$ \\
\hline 9 & .029 & .127 & B D E G Q T U V & Sonorants with high vowels (except W) \\
\hline Constant & .010 & .052 & & \\
\hline
\end{tabular}

Note-Weights for Conrad (1964) data are from stand-alone regression. For the Hull data, VAF $=89.2 \%$; for the Conrad data, VAF $=57.9 \%$. 
Table 2

Nine-cluster Alternating Least Squares Solution for the Conrad (1964) Data, Using the Initial Configuration in Table 1

\begin{tabular}{|c|c|c|c|c|}
\hline \multirow[b]{2}{*}{ Cluster } & \multicolumn{2}{|c|}{ Weight } & \multirow[b]{2}{*}{ Constituents } & \multirow[b]{2}{*}{ Interpretation } \\
\hline & $\begin{array}{c}\text { Conrad } \\
\text { Data }\end{array}$ & $\begin{array}{l}\text { Hull } \\
\text { Data }\end{array}$ & & \\
\hline 1 & .953 & .766 & $\mathbf{M} \mathbf{N}$ & Nasals \\
\hline 2 & .655 & .012 & A O & Middle vowels \\
\hline 3 & .644 & -.018 & $\mathbf{P T}$ & Unvoiced stops ending in /i/ \\
\hline 4 & .579 & .401 & F S X & Fricatives following / $/$ / \\
\hline 5 & .480 & .020 & $P Q$ & $\begin{array}{l}\text { Unvoiced stops ending in high vowels } \\
\text { (except } \mathrm{T} \text { ) }\end{array}$ \\
\hline 6 & .349 & .351 & F S & Unvoiced continuants following / $/ \mathrm{c}$ \\
\hline 7 & .306 & -.154 & $\mathrm{U} \mathbf{V}$ & Voiced continuants with high vowels \\
\hline 8 & .238 & .254 & B C D E P T & Nonsibilant stops ending in /i/ and $\mathrm{C}$ \\
\hline 9 & .151 & .166 & B D E G Q U V & $\begin{array}{l}\text { Sonorants with high vowels and } V \\
\text { (except unvoiced stops } T, P \text { ) }\end{array}$ \\
\hline Constant & .047 & .013 & & \\
\hline
\end{tabular}

Note-Clusters are ranked according to numerical weight. Weights for the Hull (1973) data are from standalone regression. For the Conrad data, VAF $=80.4 \%$; for the Hull data, VAF $=58.9 \%$.

dicating its lack of a contribution to the VAF, was quite surprising.

Because the configuration derived from the Hull data accounted for less variance in the Conrad data than expected, the latter was subjected to the alternating least squares procedure of MAPCLUS, followed by combinatorial optimization. Again, the initial configuration from Table 1 was used. In general, the clusters, shown in Table 2, were smaller than in the previous analysis. Not surprisingly, considering the above analysis, the nasals made up the most heavily weighted cluster. The middle vowels formed the second most heavily weighted cluster. Cluster 3 contained the letters with unvoiced stops ending in /i/. Cluster 4 grouped all of the fricatives. Cluster 5 contained the letters with unvoiced stops followed by high vowels, with the exception of $T$. The fact that $P$ and $Q$ were grouped together may have been caused by the similarity in voicing of their respective initial phonemes, $/ \mathrm{p} /$ and $/ \mathrm{k} /$. The absence of $\mathrm{T}$ is interpretable in light of its high-frequency noise in the plosive part of the initial phoneme as opposed to the low-frequency noise of $/ \mathrm{p} /$ and $/ \mathrm{k} /$. Cluster 6 , a subset of Cluster 4 , contained the unvoiced continuants. Cluster 7 contained the voiced continuants $/ y, v /$ with the high vowels $/ u, i /$. Cluster 8 contained the nonsibilant stops ending in the vowel $/ \mathrm{i} /$, as well as the continuant $C$. The letter $C$ was included primarily because of its confusion with $T$ and $P$. Cluster 9 contained letters with sonorant consonants followed by high vowels, and was nearly identical to Cluster 9 from Table 1. Overall, although other 9-cluster solutions offered slight improvements in fit (with VAF as high as $84 \%$ ), the clusters in Table 2 proved most interpretable.

A stand-alone regression was performed on the Hull data using, as the initial configuration, the solution derived from the Conrad data. The results are shown in Table 2. Again, one is struck by the drop in VAF from one data set to the other: from $80 \%$ for Conrad's data to only $59 \%$ for Hull's. To understand better the differences in the confusions in the two data sets, the matrices were analyzed simultaneously, using INDCLUS.

The INDCLUS analysis of the Hull (1973) and Conrad (1964) data used the clusters of Table 1 as the initial configuration. Because the initial configuration was fairly good, only one iteration of alternating least squares was specified, followed by combinatorial optimization. The two studies did not use equal numbers of subjects; there-

Table 3

INDCLUS Solution for the Hull (1973) and Conrad (1964) Data, Using the Initial Configuration From Table 1

\begin{tabular}{ccclll} 
& \multicolumn{2}{c}{ Weight } & & \\
\cline { 2 - 5 } Cluster & $\begin{array}{c}\text { Hull } \\
\text { Data }\end{array}$ & $\begin{array}{c}\text { Conrad } \\
\text { Data }\end{array}$ & \multicolumn{1}{c}{ Constituents } & \multicolumn{1}{c}{ Interpretation } \\
\hline 1 & 7.97 & 1.74 & A K & Unvoiced continuants ending in /e/ \\
2 & 6.18 & 6.81 & M N & Nasals \\
3 & 6.07 & 6.62 & F S & Unvoiced continuants following /c/ \\
4 & 4.75 & 4.52 & S X & Sibilants following / / / \\
5 & 2.36 & 0.57 & B D E V & Voiced nonsibilants ending in /i/ \\
6 & 1.63 & 2.93 & C P T & Unvoiced consonants ending in /i/ \\
7 & 1.39 & 1.49 & B D E P T & Nonsibilant stops ending in $/ \mathrm{i} /$ \\
8 & 0.68 & 0.93 & B C D E G P Q U V & Sonorants with high vowels, and C,V \\
9 & 0.11 & 4.68 & A O & High vowels \\
\hline
\end{tabular}

Note-Clusters are ranked according to numerical weight for the Hull data. Total variance accounted for is $78.6 \%$, with additive constants for Hull's and Conrad's matrices of -.253 and -.262 , respectively. 
fore, the confusion matrices were declared "matrix conditional" and normalized separately. (This option results in weights that are not directly comparable with those fitted by MAPCLUS as reported in Tables 1 and 2.) Table 3 lists the results of the analysis. Clusters 2, 3, and 4 are highly weighted for both matrices, reflecting the similarity of the confusions from the two studies. The data sets differ markedly in the weightings for Clusters 1,5 , 6 , and 9 . The results for Clusters 6 and 9 are easily explained by a hypothesis of a more degraded acoustic signal in Conrad's study, resulting in a loss of information not only about the initial unvoiced consonants (in the case of Cluster 6), but also about some vowels (in the case of Cluster 9). Less obvious, perhaps, is the possibility of explaining Clusters 1 and 5 in a similar manner. For Hull's data, $\mathrm{A}$ and $\mathrm{K}$ clustered together because they were, for the most part, confused with each other but not with other letters. Examination of the data matrices reveals that for Conrad's data, the letters $\mathrm{A}$ and $\mathrm{K}$ were confused often for $J$ as well, a voiced consonant not mistaken in Hull's study. The $(A, K)$ confusions were thus relatively less frequent in Conrad's data than in Hull's. The same type of reasoning may explain Cluster 6: The letters $C, P$, and
T were confused with other consonants ending in /i/ more often in Conrad's study than in Hull's. The INDCLUS solution accounts for more of the variance in Hull's data than in Conrad's $(84.3 \%$ vs. $72.9 \%)$.

Figures 1 and 2 summarize the results of the present analysis. Figure 1 shows the two-dimensional output from the KYST-2A nonmetric MDS procedure using Hull's data. The clusters corresponding to the derived feature list from Table 1 are circled in the figure. Figure 2 shows the corresponding two-dimensional MDS configuration for Conrad's data. The circled clusters correspond to the results of the alternating least squares approach, as summarized in Table 2. In general, the letters seem to group according to the vowel contained in them. The arrows drawn through the configurations correspond in a general sense to the position of the tongue and the part of the tongue involved in the formation of vowel sounds. These arrows are only approximations; several of the stimuli do not fit this pattern. One may find it unusual that not all of the letters appear in a cluster in the figures. The reason is that confusions among the uncircled letters accounted for very few of the total confusions in the matrix. The additive constant in the MAPCLUS solution,

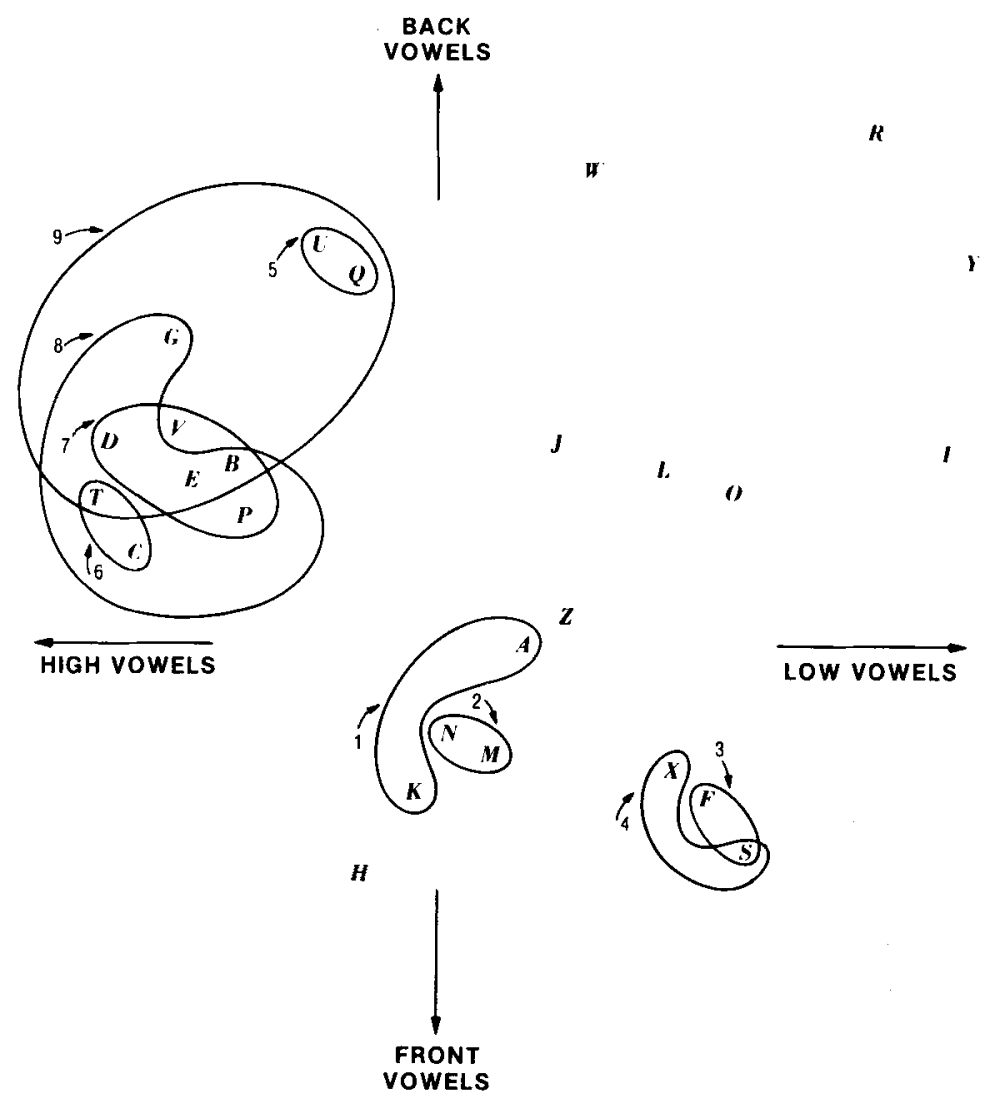

Figure 1. Two dimensional KYST-2A configuration for the Hull (1973) data. Stress is .152. Clusters are identified in Table 1. 

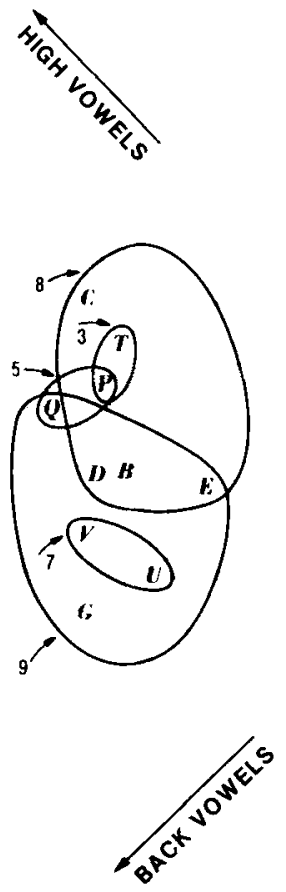

H

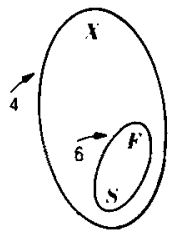

w

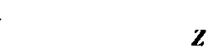

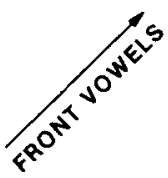

I.
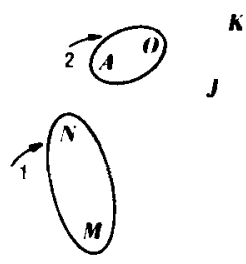

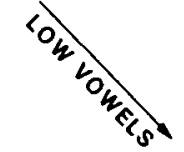

Figure 2. Two-dimensional KYST-2A configuration for the Conrad (1964) data. Stress is .190. Clusters correspond to those in Table 2.

which represents a cluster containing all 26 letters, was sufficient to represent the confusions among the uncircled letters.

\section{CONCLUSIONS}

In the present study, an attempt was made to explain the underlying patterns of confusions in auditorially presented alphabets using phonetic features. The initial analysis, which used the feature list based upon the work of Wickelgren (1966), did not prove successful, probably because the stimuli used in Wickelgren's study were single consonant phonemes. The stimuli in the present study, on the other hand, were composed of several phonemes. In an earlier study, Wickelgren (1965) found that the most often confused alphabet letter pairs share at least one common phoneme. This observation tended, to some degree, to be true of Hull's (1973) and Conrad's (1964) data in the present study. One finding that emerged from the present study was that not all letters that share a phoneme are confused. The letters $\mathrm{H}$ and $\mathrm{J}$, for example, which share the phoneme /e/, were rarely confused in Hull's and Conrad's studies. It seems not only that the letters must share the same phoneme, but that the phoneme must occupy the same position in the stimulus name.

The clusters obtained in the present study for both sets of data indicate that the vowel sounds present in the letters contribute substantially to the confusions between them. This condition holds true for cases in which the vowel comes at the end of the letter name and also for those in which it is in the initial position. The confusions between $\mathrm{M}$ and $\mathrm{N}$ and among $\mathrm{F}, \mathrm{S}$, and $\mathrm{X}$ illustrate the importance of the vowel sound. The phonetic features of the consonants also play a role in the determination of letter similarities. Usually, the consonants in highly confusable letters have one or more acoustic features in common, especially in Hull's data set.

It was possible to construct, on the basis of the phonetic features of the letters, a feature list that accounted for $89 \%$ of the variance in the Hull data. When applied to the Conrad's data set, this feature list accounted for only $58 \%$ of the variance. This drop in VAF is much greater than one would expect from shrinkage alone. A more optimum clustering representation of Conrad's data resulted in over $80 \%$ of the variance accounted for. When this cluster solution was applied to Hull's data, the VAF was only $59 \%$.

The weaker performance of each feature list when applied to the data set other than that upon which the solution was based, as well as for the differential weightings of the clusters in the INDCLUS solution, led to the conclusion that the data sets are not readily substitutable. One possible cause of the differences could be Conrad's use of untrained speakers for the presentation of the stimuli. It is possible, too, that the amount of background noise in the Conrad study was slightly higher than in the Hull study. In any event, the increased number of confusions and the subtle changes in the patterns of the confusions 
in Conrad's data appear indicative of a degraded acoustic signal. One manifestation of this trend may be seen in the overall pattern of confusions in the two data sets. In the clusters in Table 1, all but the last cluster contain letters that share a common vowel. In Table 2 , which lists the cluster solution for Conrad's data, several of the clusters contain letters whose vowels are not identical, but which do share a common articulatory characteristic. Evidently, the subtle differences in the auditory signals have been masked, presumably by extraneous noise. It is not surprising, then, that the confusions in the Conrad data differ from, and are less interpretable than those in Hull's data set. Despite these differences, however, the patterns of the clusters are similar across the data sets, as the weightings of the clusters in the INDCLUS solution affirm. Thus, it appears that there are fundamental attributes of the letters which lead to the confusions among them.

\section{REFERENCES}

Arabie, P., \& Carroll, J. D. (1980a). How to use MAPCLUS, a computer program for fitting the ADCLUS model. Murray Hill, NJ: AT\&T Bell Laboratories (Room 2F-128A).

Arabie, P., \& Carroll, J. D. (1980b). MAPClUS: A mathematical programming approach to fitting the ADCLUS model. Psychometrika, 45, 211-235.

Arabie, P., Carroll, J. D., \& DeSarbo, W. S. (1987). Three-way scaling and clustering. Newbury Park, CA: Sage.

ARABIE, P., \& Soli, S. D. (1982). The interface between the type of regression and methods of collecting proximities data. In R. Golledge \& J. N. Rayner (Eds.), Proximity and preference: Problems in the multidimensional analysis of large data sets (pp. 90-115). Minneapolis: University of Minnesota Press.

Carroll, J. D., \& ARabie, P. (1982). How to use INDCLUS, a computer program for fitting the individual differences generalization of the ADCLUS model. Murray Hill, NJ: AT\&T Bell Laboratories.

CARroll, J. D. , A ARABIE, P. (1983). INDCLUS: An individual differences generalization of the ADCLUS model and the MAPCLUS algorithm. Psychometrika, 48, 157-164.

Carroll, J. D., \& Chang, J. J. (1970). Analysis of individual differences in multidimensional scaling via an $N$-way generalization of 'Eckart-Young' decomposition. Psychometrika, 35, 283-319.

Chang, J.-J., \& ShEPARD, R. N. (1966, April). Exponential fitting in the proximity analysis of confusion matrices. Paper presented at the annual meeting of the Eastern Psychological Association, New York.

ConRad, R. (1964). Acoustic confusions in immediate memory. British Journal of Psychology, 55, 75-84.

HUBERT, L. J., \& BAKER, F. B. (1977). Analyzing distinctive features. Journal of Educational Statistics, 2, 79-98.
HUberT, L. J., \& BAKER, F. B. (1979). Evaluating the symmetry of a proximity matrix. Quality \& Quantity, 13, 77-84.

HuLl, A. J. (1973). A letter-digit matrix of auditory confusions. British Journal of Psychology, 64, 579-585.

JARDINE, N., \& SiBSON, R. (1968). The construction of hierarchic and non-hierarchic classifications. Computer Journal, 11, 177-184.

Johnson, S. C. (1967). Hierarchical clustering schemes. Psychometrika, 32, 241-254.

KEWLEY-PORT, D. (1983). Time-varying features as correlates of place of articulation in stop consonants. Journal of the Acoustical Society of America, 73, 322-335.

KlatT, D. H. (1968). Structure of confusions in short-term memory between English consonants. Journal of the Acoustical Society of America, 44, 401-407.

LaNCE, G. N., \& Williams, W. T. (1967). A general theory of classificatory sorting strategies. I. Hierarchical systems. Computer Joumal, 9, 373-380.

Miller, G. A., \& NiCELY, P. E. (1955). An analysis of perceptual confusions among some English consonants. Journal of the Acoustical Society of America, 27, 338-352.

Morgan, B. J. T. (1973). Cluster analyses of two acoustic confusion matrices. Perception \& Psychophysics, 13, 13-24.

ShePard, R. N. (1972). Psychological representation of speech sounds. In E. E. David \& P. B. Denes (Eds.), Human communication: $A$ unified view (pp. 67-113). New York: McGraw-Hill.

SHEPARD, R. N. (1974). Representation of structure in similarity data: Problems and prospects. Psychometrika, 39, 373-421.

Shepard, R. N., \& ARABIE, P. (1979). Additive clustering: Representation of similarities as combinations of discrete overlapping properties. Psychological Review, 86, 87-123.

Soli, D. D., Arabie, P., \& Carroll, J. D. (1986). Discrete representation of perceptual structure underlying consonant confusions. Journal of the Acoustical Society of America, 79, 826-837.

STEVENS, K. N. (1972). The quantal nature of speech: Evidence from articulatory-acoustic data. In E. E. David \& P. B. Denes (Eds.), Human communication: A unified view (pp. 51-66). New York: McGraw-Hill.

VAN DER KAMP, L. J., \& Pols, L. C. W. (1971). Perceptual analysis from confusions between vowels. Acta Psychologica, 35, 64-77.

WANG, M. D., \& BLGER, R. C. (1973). Consonant confusions in noise: A study of perceptual features. Journal of the Acoustical Society of America, 54, 1248-1266.

WiCKELGREN, W. A. (1965). Acoustic similarity and intrusion errors in short-term memory. Journal of Experimental Psychology, 70, 102-108.

WiCKelgren, W. A. (1966). Distinctive features and errors in shortterm memory for English consonants. Journal of the Acoustical Society of America, 39, 388-398.

WoLD, H. (1966). Estimation of principal components and related models by iterative least squares. In P. R. Krishnaiah (Ed.), Multivariate analysis (pp. 391-420). New York: Academic Press.

(Manuscript received January 19, 1988; revision accepted for publication September 30, 1988.) 